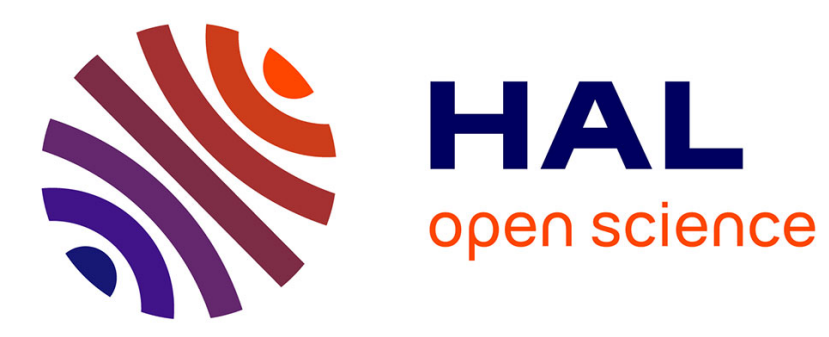

\title{
Chaotic advection at large Péclet number: Electromagnetically driven experiments, numerical simulations, and theoretical predictions
}

Aldo Figueroa, Patrice Meunier, Sergio Cuevas, Emmanuel Villermaux, Eduardo Ramos

\section{To cite this version:}

Aldo Figueroa, Patrice Meunier, Sergio Cuevas, Emmanuel Villermaux, Eduardo Ramos. Chaotic advection at large Péclet number: Electromagnetically driven experiments, numerical simulations, and theoretical predictions. Physics of Fluids, 2014, 26 (1), pp.013601. 10.1063/1.4861004 . hal01174033

\section{HAL Id: hal-01174033 \\ https://hal.science/hal-01174033}

Submitted on 8 Jul 2015

HAL is a multi-disciplinary open access archive for the deposit and dissemination of scientific research documents, whether they are published or not. The documents may come from teaching and research institutions in France or abroad, or from public or private research centers.
L'archive ouverte pluridisciplinaire HAL, est destinée au dépôt et à la diffusion de documents scientifiques de niveau recherche, publiés ou non, émanant des établissements d'enseignement et de recherche français ou étrangers, des laboratoires publics ou privés. 


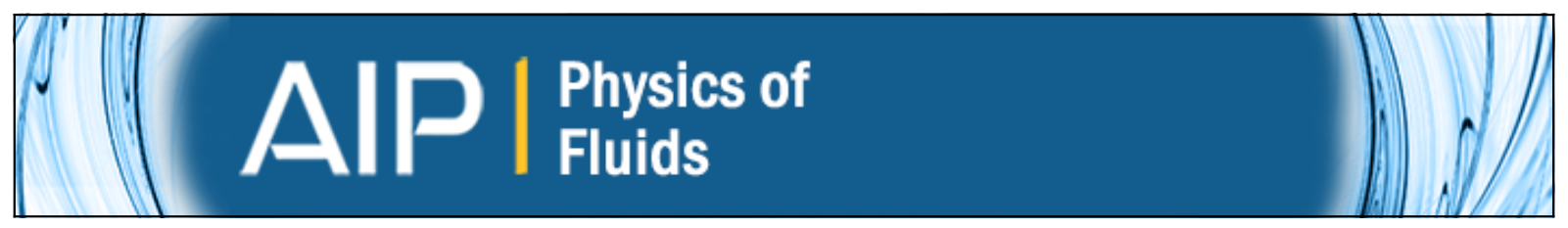

Chaotic advection at large Péclet number: Electromagnetically driven experiments, numerical simulations, and theoretical predictions

Aldo Figueroa, Patrice Meunier, Sergio Cuevas, Emmanuel Villermaux, and Eduardo Ramos

Citation: Physics of Fluids (1994-present) 26, 013601 (2014); doi: 10.1063/1.4861004

View online: http://dx.doi.org/10.1063/1.4861004

View Table of Contents: http://scitation.aip.org/content/aip/journal/pof2/26/1 ?ver=pdfcov

Published by the AIP Publishing

\section{$\underset{\substack{\text { Aubloling } \\ \text { pulp }}}{A}$ Re-register for Table of Content Alerts}

\section{Create a profile.}




\title{
Chaotic advection at large Péclet number: Electromagnetically driven experiments, numerical simulations, and theoretical predictions
}

\author{
Aldo Figueroa, ${ }^{1}$ Patrice Meunier, ${ }^{2}$ Sergio Cuevas, ${ }^{3}$ Emmanuel Villermaux, ${ }^{2}$ \\ and Eduardo Ramos ${ }^{3}$ \\ ${ }^{1}$ Facultad de Ciencias, Universidad Autónoma del Estado de Morelos, Cuernavaca, \\ Morelos 62209, Mexico \\ ${ }^{2}$ Aix-Marseille Univ., CNRS, Centrale Marseille, IRPHE, Marseille F-13384, France \\ ${ }^{3}$ Instituto de Energías Renovables, Universidad Nacional Autónoma de México, \\ A.P. 34, Temixco, Morelos 62580, Mexico
}

(Received 29 April 2013; accepted 22 November 2013; published online 15 January 2014)

\begin{abstract}
We present a combination of experiment, theory, and modelling on laminar mixing at large Péclet number. The flow is produced by oscillating electromagnetic forces in a thin electrolytic fluid layer, leading to oscillating dipoles, quadrupoles, octopoles, and disordered flows. The numerical simulations are based on the Diffusive Strip Method (DSM) which was recently introduced (P. Meunier and E. Villermaux, "The diffusive strip method for scalar mixing in two-dimensions," J. Fluid Mech. 662, 134-172 (2010)) to solve the advection-diffusion problem by combining Lagrangian techniques and theoretical modelling of the diffusion. Numerical simulations obtained with the DSM are in reasonable agreement with quantitative dye visualization experiments of the scalar fields. A theoretical model based on log-normal Probability Density Functions (PDFs) of stretching factors, characteristic of homogeneous turbulence in the Batchelor regime, allows to predict the PDFs of scalar in agreement with numerical and experimental results. This model also indicates that the PDFs of scalar are asymptotically close to log-normal at late stages, except for the large concentration levels which correspond to low stretching factors. (c) 2014 AIP Publishing LLC. [http://dx.doi.org/10.1063/1.4861004]
\end{abstract}

\section{INTRODUCTION}

Fluid mixing is an important subject for multiple applications in geophysics, chemistry, and biology, as well as in many relevant industrial devices. Over the years, significant research efforts have been devoted to find methods to quantify and accelerate mixing. Some methods take advantage of mechanical interactions to mix fluids, for instance, by turning a propeller in a container or through the periodic displacement of the container's walls. ${ }^{1}$ The mixing obtained in the annular region between rotating concentric cylinders has also received attention. ${ }^{2}$ Of particular interest are nonintrusive methods that rely on the use of electromagnetic forces to produce stirring and mixing in electrically conducting fluids. In fact, electromagnetic forcing has been widely used experimentally to produce mixing in shallow layers of liquid metals ${ }^{3}$ or electrolytes. ${ }^{4-6}$ The basic idea is to generate a rotational Lorentz force in a thin layer of a conducting fluid by the interaction of electric currents with a steady external magnetic field. As a matter of fact, under certain conditions these flows may present a quasi-two-dimensional (Q2D) behavior. ${ }^{7-9}$ Incidentally, Q2D flows display some similarities with those found in the ocean and the atmosphere ${ }^{10}$ where the transport of particles ${ }^{11}$ or nutrients ${ }^{12}$ is of great importance.

While turbulent flows lead to fast mixing, it is also known that mixing can be substantially enhanced in laminar flows by subjecting the fluid to an appropriate chaotic kinematics. ${ }^{13}$ Dynamical system approaches based on flow kinematics have provided a first insight into chaotic mixing. ${ }^{13-15}$ Extensive studies have yielded important insights into the geometric structures that govern the 
mixing process, particularly in two-dimensional flows. A central goal of the analysis of fluid mixing is to understand how an initially inhomogeneous fluid concentration is homogenized and predict the rate at which this process takes place. This can be done, for instance, by following how typical scalar concentration fluctuations decay with respect to the mean. ${ }^{16}$ Numerous studies have been devoted to this question and, in chaotic flows where material lines grow exponentially in time, a global exponential decay of the dye's spatial contrast is observed. ${ }^{17,18}$ When the flow is not chaotic and dominated by sustained shear, ${ }^{19,20}$ presents elliptic islands, ${ }^{21}$ or in slow motion regions like walls $^{22}$ the decay is slowed down, and may be algebraic in time (see Villermaux ${ }^{23}$ for a review of the different scenarios and applications).

However, a complete description of the scalar field requires the knowledge of its concentration distribution, or probability density function (PDF) $P(c)$ of the concentration levels $c$ of the substance being mixed, and not only of an index function of its two first moments. The shape, and temporal evolution of the PDF, is also indicative of the underlying processes directing the mixture toward uniformity. ${ }^{20,24}$ That shape is typically bimodal at early stages, exhibiting two peaks (one at zero, the concentration of the diluting medium, the other at the initial concentration of the dye) and a single peak at late stages (at the average concentration). Understanding the kinetics and nature of the transition between these two extremes is the subject of mixing.

The importance of advection relative to molecular diffusion of a scalar in a given mixing problem is measured by a dimensionless parameter called the Péclet number, defined as $P e=U L / D$, where $U$ is a typical flow speed, $L$ is a characteristic length scale, and $D$ is the molecular diffusivity. Physically, the condition $P e \gg 1$ implies that the stretching of the fluid interface by advection proceeds for a substantial amount of time before diffusive effects eventually smear out the interface and mix the fluids at small length scales. Chaotic mixing devices aim to achieve exponential growth of the total interface length in time by stretching and folding the fluid in similar fashion to the transformation maps of chaotic dynamics theory. ${ }^{25}$ However, large $P e$ values also render the numerical solution of the advection-diffusion equation extremely challenging. ${ }^{26}$ Recently, a new numerical method for the analysis of scalar mixing in two-dimensional (2D) advection fields, namely the diffusive strip method (DSM), was introduced by Meunier and Villermaux. ${ }^{24}$ The DSM is able to solve the full advection-diffusion problem, including the smoothing and decaying of the scalar, although it uses a Lagrangian technique to define the location of the scalar. This method is thus intermediate between the Eulerian resolution of the equations on a mesh (which is limited to small Péclet number) and the Lagrangian advection of non-diffusive tracers (which corresponds to an infinite Péclet number). The DSM combines the advantages of both techniques and is thus valid for all Péclet numbers, including very large ones.

Different strategies have been devised to increase mixing rates in electromagnetically driven flows, for instance to use a disordered array of magnets, ${ }^{4}$ alternating currents,${ }^{27}$ or sufficiently strong direct currents. ${ }^{28}$ Although increasing the mixing rates is evidently an important topic, the present contribution is not focused in developing an optimized mixer but rather at providing experimental results that can be compared with numerical simulations based on the DSM. With this aim, we analyze experimentally and theoretically the mixing obtained in flows of oscillating vortices in a shallow layer of an electrolyte driven by electromagnetic forces produced by the interaction of an alternating current and the steady magnetic field of one or several dipole magnets. Although three-dimensional effects may appear in these flows ${ }^{29}$ the present experiments were carried out under conditions where a quasi-two-dimensional behavior prevails $s^{8,9}$ and, therefore, are particularly suited for comparison with numerical results based on DSM. We start with a brief explanation of the experimental and numerical procedure. The results are presented and analyzed in Sec. IV and followed by the conclusions.

\section{EXPERIMENTAL PROCEDURE}

The experimental set-up was already described in detail in previous studies. ${ }^{7,8}$ Essentially, dyed water visualization experiments were carried out in multipolar flows driven by electromagnetic forces where a one-directional alternating current is applied in a fluid layer $4 \mathrm{~mm}$ thick of a weak electrolytic solution of sodium bicarbonate $\left(\mathrm{NaHCO}_{3}\right.$ at $8.6 \%$ by weight) in the presence of one 
or several permanent magnets. The flow takes place in a rectangular container $(28 \mathrm{~cm} \times 36 \mathrm{~cm} \times$ $1.6 \mathrm{~cm}$ ) where a pair of parallel electrodes are connected to a Stanford Research System DS345 function generator that allows to modify the frequency and the phase of the signal. The amplitude of the electric current was fixed to $80 \mathrm{~mA}$ with frequency of $f=50 \mathrm{mHz}$. Arrays of rectangular parallelepiped magnets with a side length of $25.4 \mathrm{~mm}$, height of $12.7 \mathrm{~mm}$, and maximum strength of $0.38 \mathrm{~T}$ were placed underneath the bottom wall. Depending on the number of magnets and their geometrical position, the Lorentz force generated different oscillating vortical flow structures in the $(x-y)$ plane. In order to analyze the mixing properties of the electromagnetically driven flows, a uniform blob of dye $\left(V \approx 30 \mathrm{~mm}^{3}\right.$ ) was deposited on the electrolyte layer on top of the magnets zone where a high stretching region exists. The dyed liquid forms a circular blob with radius $r \approx 5 \mathrm{~mm}$ and thickness of approximately $0.4 \mathrm{~mm}$. The dye was disodium fluorescein with initial concentration $c_{0} \approx 10^{-5} \mathrm{~mol}^{-1}$ and molecular diffusivity $D=5 \times 10^{-10} \mathrm{~m}^{2} / \mathrm{s}$. The dyed blob stays at the free surface probably due to weak thermal effects and follows the motion of the fluid. Although there is diffusion in the vertical direction (which decreases the dye concentration), this effect does not lead to a decrease of the intensity measured by the camera which integrates over the whole depth the light emitted by the dye. Indeed, the dye is illuminated in volume using two symmetrically located ultraviolet lamps in an otherwise dark room. The lamps are far enough to create a variation of light between the center and the side of the image smaller than $9 \%$. Moreover, as the electrolyte is a transparent medium, the bottom wall was covered with a black sheet for ensuring the contrast in the visualization. Images of the fluorescein's concentration field were recorded every $5 \mathrm{~s}$ with a Nikon D80 camera with a AF micro-nikkor $60 \mathrm{~mm} \mathrm{f} / 2.8 \mathrm{D}$ lens. The actual area of the captured image is $34 \mathrm{~cm} \times 23 \mathrm{~cm}$. With these parameters, the pixel size is $8.8 \times 10^{-5} \mathrm{~m}$ which is of the same order of the Batchelor scale $\left(7 \times 10^{-5} \mathrm{~m}\right)$. The resulting background subtracted grey levels are proportional to the dye concentration.

In the absence of fluorescein, the maximum velocity that was reached in the experiments is $U$ $=1.4 \times 10^{-2} \mathrm{~m} / \mathrm{s}$, which corresponds to an injected steady direct current of $80 \mathrm{~mA} .^{7}$ With this velocity scale, the maximum Reynolds number based on the magnet side length $(L=25.4 \mathrm{~mm})$, kinematic viscosity $\left(v=10^{-6} \mathrm{~m}^{2} / \mathrm{s}\right),(R e=U L / v)$ is equal to $R e=350$. In turn, the oscillation Reynolds number $\left(R_{\omega}=\omega L^{2} / \nu\right)$ based on the experimental forcing frequency $(\omega=2 \pi f, f=50 \mathrm{mHz})$ corresponds to $R_{\omega}=200$. Note that the maximum Reynolds number based on the layer thickness ( $h=4 \mathrm{~mm})\left(R e_{h}=U h / v\right)$ is equal to $R e_{h}=55$. Considering the molecular diffusivity $D=5 \times$ $10^{-10} \mathrm{~m}^{2} \mathrm{~s}^{-1}$, the Péclet number is equal to $P e=7 \times 10^{5}$. It should be noted that in the present experiments (and corresponding simulations) a maximum current amplitude of $60 \mathrm{~mA}$ was reached, the smaller amplitude being attributed to a decrease of the effective electrical conductivity of the electrolyte caused by the presence of fluorescein.

These values are much smaller from those used in experiments where 3D effects were observed. ${ }^{29}$ The flow is thus calculated numerically by a quasi-2D model, which has been validated by experimental velocity fields of multipolar flows with electrical current amplitudes up to $80 \mathrm{~mA} .^{9}$

\section{NUMERICAL MODEL}

\section{A. Quasi-two-dimensional numerical model for the flow}

The velocity fields of the explored experimental flows were numerically simulated using the Q2D model presented in previous works. ${ }^{7-9}$ The Q2D model is based on averaging the balance equations in the normal direction so that flows are described in terms of two-dimensional core variables with a linear friction that accounts for the effects of the oscillating boundary layer. The model also considers the decay of the magnetic field in the direction normal to the fluid layer. The coordinate system is placed at the bottom wall of the container in such a way that $x-y$ planes remain parallel to this wall and the normal coordinate points in the $z$-direction. In dimensionless terms, the averaged governing equations read

$$
\frac{\partial u}{\partial x}+\frac{\partial v}{\partial y}=0
$$




$$
\begin{gathered}
R_{\omega} \frac{\partial u}{\partial t}+u \frac{\partial u}{\partial x}+v \frac{\partial u}{\partial y}=-\frac{\partial p}{\partial x}+\nabla_{\perp}^{2} u-\frac{u}{\tau} \\
R_{\omega} \frac{\partial v}{\partial t}+u \frac{\partial v}{\partial x}+v \frac{\partial v}{\partial y}=-\frac{\partial p}{\partial y}+\nabla_{\perp}^{2} v-\frac{v}{\tau}-\alpha \operatorname{Re}_{*} \mathcal{B}_{z}^{0} \sin (t),
\end{gathered}
$$

where $u$ and $v$ stand for the velocity components in the $x$ - and $y$-direction, respectively, normalized by $u_{0}=v / L, v$ and $L$ being the kinematic viscosity of the fluid and the magnet characteristic length. The pressure field is denoted by $p$, normalized by $\rho u_{0}^{2}$, where $\rho$ is the density of the fluid. Coordinates $x$ and $y$ are normalized by $L$, while coordinate $z$ is normalized by the depth of the layer $h$. In turn, time $t$ is normalized by $\omega$, where $\omega=2 \pi f$ is the angular frequency and $f$ is the ordinary frequency of the electrical current. The last term on the right-hand side of Eq. (3) considers the oscillating Lorentz force created by the non-uniform magnetic field distribution $\mathcal{B}_{z}^{0}(x, y)$, and the applied alternating current in the $x$-direction, namely, $\sin (t) \hat{\boldsymbol{x}}$, which is normalized by the current amplitude $j^{0}$. Here, $\mathcal{B}_{z}^{0}$ that is normalized by the maximum magnetic field strength represents the non-uniformity of the field in the $(x-y)$ plane at a given height $z$ using an analytical expression for the field of a magnetized rectangular surface uniformly polarized in the normal direction. ${ }^{8,30,31}$ The sub-index $\perp$ denotes the projection of the $\nabla^{2}$ operator on the $(x-y)$ plane. The parameter $\operatorname{Re}_{*}=U_{0} L / v$ stands for the Reynolds number based on the characteristic bulk velocity $U_{0}=j^{0} B_{\max } L^{2} / \rho \nu$, which is obtained from a balance between viscous and Lorentz forces. However, this balance only considers lateral viscous diffusion and does not take into account the friction at the bottom wall. Therefore, $R e_{*}$ does not coincide with the experimental Reynolds number based on the maximum velocity. Note that both Eqs. (2) and (3) contain the linear friction term, called Rayleigh friction, which involves a characteristic dimensionless time scale, $\tau$, for the damping of vorticity due to dissipation in the viscous layers at the bottom wall, and whose inverse is given by

$$
\tau^{-1}=\Re\left\{\frac{a \sinh \left(\sqrt{a} \epsilon^{2}\right)+\sqrt{a} \beta\left(e^{-\beta \epsilon^{2}}-\cosh \left(\sqrt{a} \epsilon^{2}\right)\right)}{\sinh \left(\sqrt{a} \epsilon^{2}\right)-\frac{\beta e^{-\beta \epsilon^{2}}}{\sqrt{a}}\left(\cosh \left(\sqrt{a} \epsilon^{2}\right)-1\right)+\frac{\sqrt{a}}{\beta} \cosh \left(\sqrt{a} \epsilon^{2}\right)\left(e^{-\beta \epsilon^{2}}-1\right)}\right\},
$$

where $a=i R_{\omega}, \varepsilon=h / L$ is the aspect ratio that compares the characteristic lengths in the normal and transversal directions and $\mathfrak{R}$ indicates the real part of the expression inside the brackets \{\} . According to the shallow flow approximation, $\varepsilon$ is assumed to be less than unity (in the experiment, the maximum value of $\varepsilon$ is 0.16 ). The parameter $\tau^{-1}$ comes out, after integration in the $z$-direction, from considering that the viscous layers at the bottom wall behave as a Stokes velocity profile. ${ }^{8}$ Further, due to the decay of the magnetic field in the normal direction, the Lorentz force term in Eq. (3) includes the damping factor $\alpha$ given by ${ }^{8}$

$$
\alpha=\int_{0}^{\varepsilon} \exp (-\beta \varepsilon z) d z
$$

where $\beta=2.16$ was obtained from experimental measurements. The system of Eqs. (1)-(3), along with the friction model (Eq. (4)), the factor $\alpha$ (Eq. (5)), and the $2 \mathrm{D}$ distribution of the magnetic field $\mathcal{B}_{z}^{0}$, was solved numerically using a finite difference code based on Griebel et al. ${ }^{32}$ extended for magnetohydrodynamic flows. The numerical code considers the whole experimental domain and assumes that velocity components satisfy non-slip conditions at the boundaries of the container. The numerical velocity fields were used to calculate the Lagrangian trajectories as well as to feed the advection-diffusion equation for a passive scalar which was solved by using the diffusive strip method explained below.

\section{B. Diffusive strip method}

The central question in scalar mixing is the satisfactory description of the spatial distribution of the concentration $c$ of a substance being mixed, and of the corresponding concentration content of the field. The concentration $c$, which acts as a passive scalar, satisfies the advection-diffusion 
equation which is expressed in dimensionless terms as

$$
R_{\omega} \frac{\partial c}{\partial t}+(\boldsymbol{u} \cdot \nabla) c=\frac{R e_{*}}{P e} \nabla^{2} c,
$$

where the previously introduced Péclet number, $P e$ (based on the characteristic velocity $U_{0}$ and the magnet characteristic length $L$ ), gives the ratio of convective and diffusive effects. In water, the diffusivity of the scalar is usually much smaller than the kinematic diffusivity (by 3 orders of magnitude for fluorescein), such that even for a moderate Reynolds number the Péclet number is very large. As a consequence, the scalar is stretched into long and thin filaments. This imposes a very fine mesh in the numerics in order to resolve the concentration gradients. All numerical techniques solving the advection-diffusion equation on a mesh are thus highly demanding in memory. We thus use a new numerical method, the diffusive strip method, which has been introduced in order to solve 2D mixing problems at large Péclet numbers. ${ }^{24}$ This method is based on Lagrangian techniques, since the scalar is introduced as a material strip (or filament) represented by an array of passive tracers whose positions $\left(\mathbf{x}_{i}\right)$ are computed by integrating the kinematic equation of motion

$$
R_{\omega} \frac{d \boldsymbol{x}_{i}}{d t}=\boldsymbol{u}_{i}
$$

where $\boldsymbol{u}_{i}$ is the flow velocity at the position of the $i$ th tracer. The key point of the method is that the diffusion of the filament can be predicted theoretically if the thickness of the diffusing strip is smaller than its local radius of curvature. Indeed, since the stretching rate of the filament is known (from the distance between two consecutive tracers), the profile of concentration as a function of the normal coordinate $n$ is Gaussian and given at time $t$ by ${ }^{24}$

$$
c(n)=\frac{1}{\sqrt{1+4 \xi_{i}}} e^{-n^{2} / s_{i}^{2}\left(1+4 \xi_{i}\right)} .
$$

Here, the dimensionless time $\xi_{i}$ and the striation thickness $s_{i}$ can be calculated numerically from

$$
\frac{d \xi_{i}}{d t}=\frac{R e}{R_{\omega} P e} \frac{1}{s_{i}^{2}}, \quad s_{i}=s_{0} \Delta x_{i}^{0} / \Delta x_{i},
$$

where $s_{0}$ is the initial thickness of the strip, $\Delta x_{i}$ is the separation between consecutive tracers, and $\Delta x_{i}^{0}$ is the initial separation. A Fortran code was developed to implement the DSM. Positions of the particles are calculated from Lagrangian tracking by integrating Eq. (7) with a second order approximation. A detailed explanation of the numerical procedure for the reconstruction of the 2D scalar field (Eq. (10)) as a function of time (Eq. (9)), along with the cusps treatment, can be found in the paper by Meunier and Villermaux. ${ }^{24}$ The concentration field $c$ is reconstructed by adding small Gaussian ellipses centered on each tracer, namely, ${ }^{24}$

$$
c=\sum_{i} \frac{1 / 1.77264}{\sqrt{1+4 \xi_{i}}} \exp \left(-\frac{\left[\left(\boldsymbol{x}-\boldsymbol{x}_{i}\right) \cdot \widehat{\sigma}_{i}\right]^{2}}{\Delta x_{i}^{2}}-\frac{\left[\left(\boldsymbol{x}-\boldsymbol{x}_{i}\right) \cdot \widehat{n}_{i}\right]^{2}}{s_{i}^{2}\left(1+4 \xi_{i}\right)}\right) .
$$

The succession of ellipses forms a filament that represents the scalar distribution. In Eq. (10), the term $s_{i}^{2}\left(1+4 \xi_{i}\right)$ is the local width of the filament and $\Delta x_{i}$ is the distance between two consecutive tracers. The local tangent and normal unitary vectors are denoted by $\widehat{\sigma}_{i}$ and $\widehat{n}_{i}$, respectively. The numerical constant 1.77264 is due to the overlap of consecutive ellipses.

The DSM has been previously validated with the Lamb-Oseen vortex flow, for which a theoretical prediction exists. ${ }^{19}$ This method is extremely efficient for large Péclet numbers since it only calculates the position and dimensionless time of each tracer, such that the filament is described by three 1D arrays. This is much less at early stages than the 2D array needed to describe the whole concentration field on a mesh. Moreover, the number of tracers is independent of the thickness of the filament and this technique can thus be used for any Péclet number, including very large ones. However, in this method the filament has to be refined by adding new tracers, whose number increases exponentially by the flow kinematics, thus limiting the duration of the simulation for the same trivial storage capacity reason. 


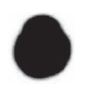

(a)

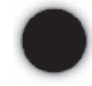

(b)

FIG. 1. Initial condition for the experiment (a) and for the numerical DSM simulation (b). The dimension of the images is approximately $4 \times 4 \mathrm{~cm}^{2}$.

\section{RESULTS}

\section{A. Scalar fields}

We first compare the numerical results of the scalar fields calculated by the diffusive strip method with electromagnetically driven vortex flows. Experimentally, a blob of dye with uniform concentration is initially located at $(x \approx 0, y \approx 0)$ as shown in Fig. 1(a). Numerically, the strip is initiated on a circle of radius $r=0.5 \mathrm{~cm}$ with a large thickness $s_{0}=4 \mathrm{~cm}$. The overlap of both sides of the strip fills the center of the circle with a nearly uniform concentration, as shown in Fig. 1(b).

Figure 2 shows the temporal evolution of the scalar. As time increases, the tracer is advected periodically toward positive and negative $y$ by the oscillating vortex dipole. Every half-cycle, the scalar is pushed away from the magnet zone forming a kind of semicircles around the central zone. There is a very good agreement between the numerics and the experiment on the position of the scalar. This underlines the fact that the quasi-2D model is able to reproduce the flow well.
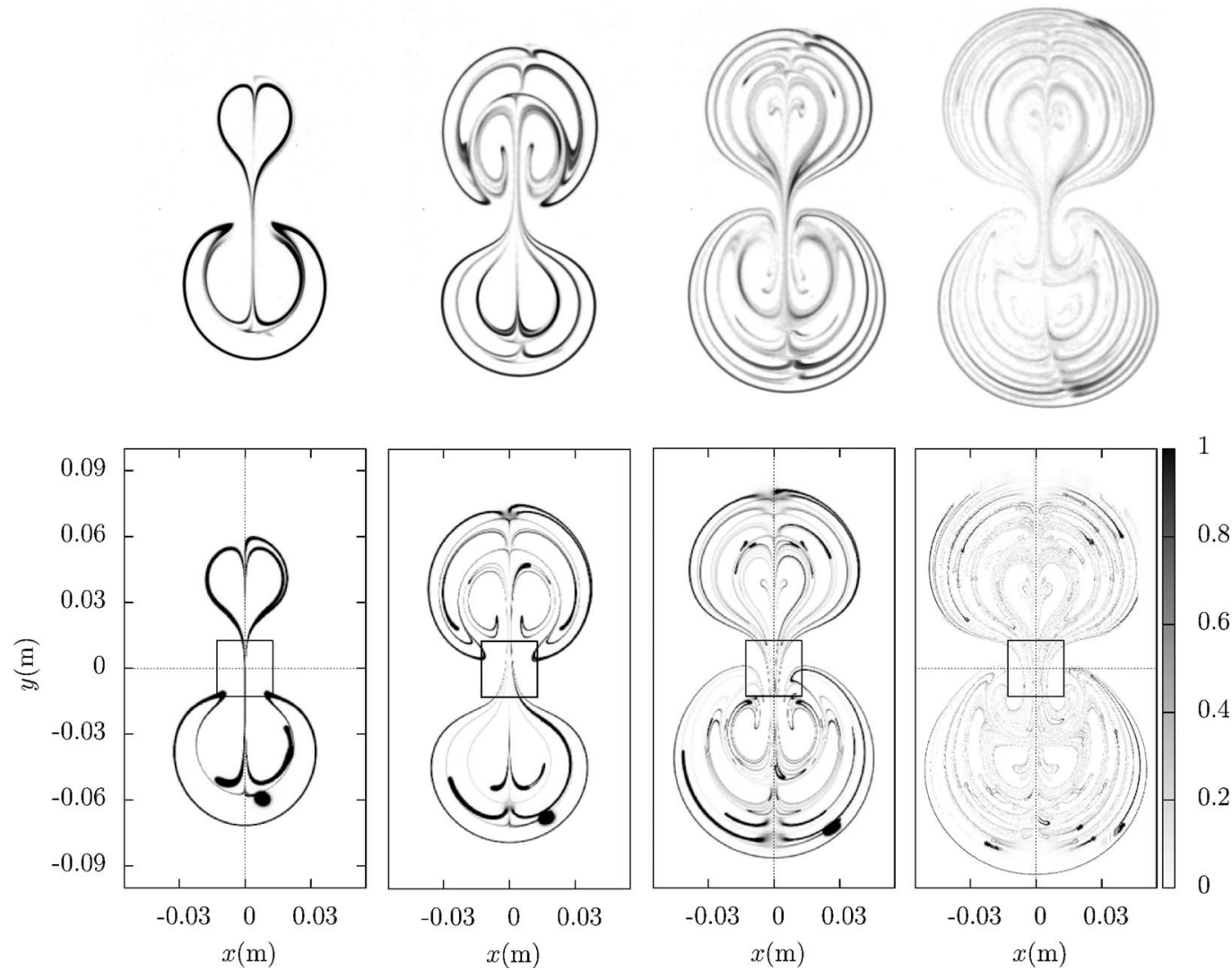

FIG. 2. Oscillating dipole vortex flow. First row, experimental visualization; second row, numerical simulation. First column, $t=30 \mathrm{~s}$; second column, $t=60 \mathrm{~s}$; third column, $t=90 \mathrm{~s}$; fourth column, $t=150 \mathrm{~s}$. The square denotes the footprint of the magnet polarized in the positive $z$-direction. 

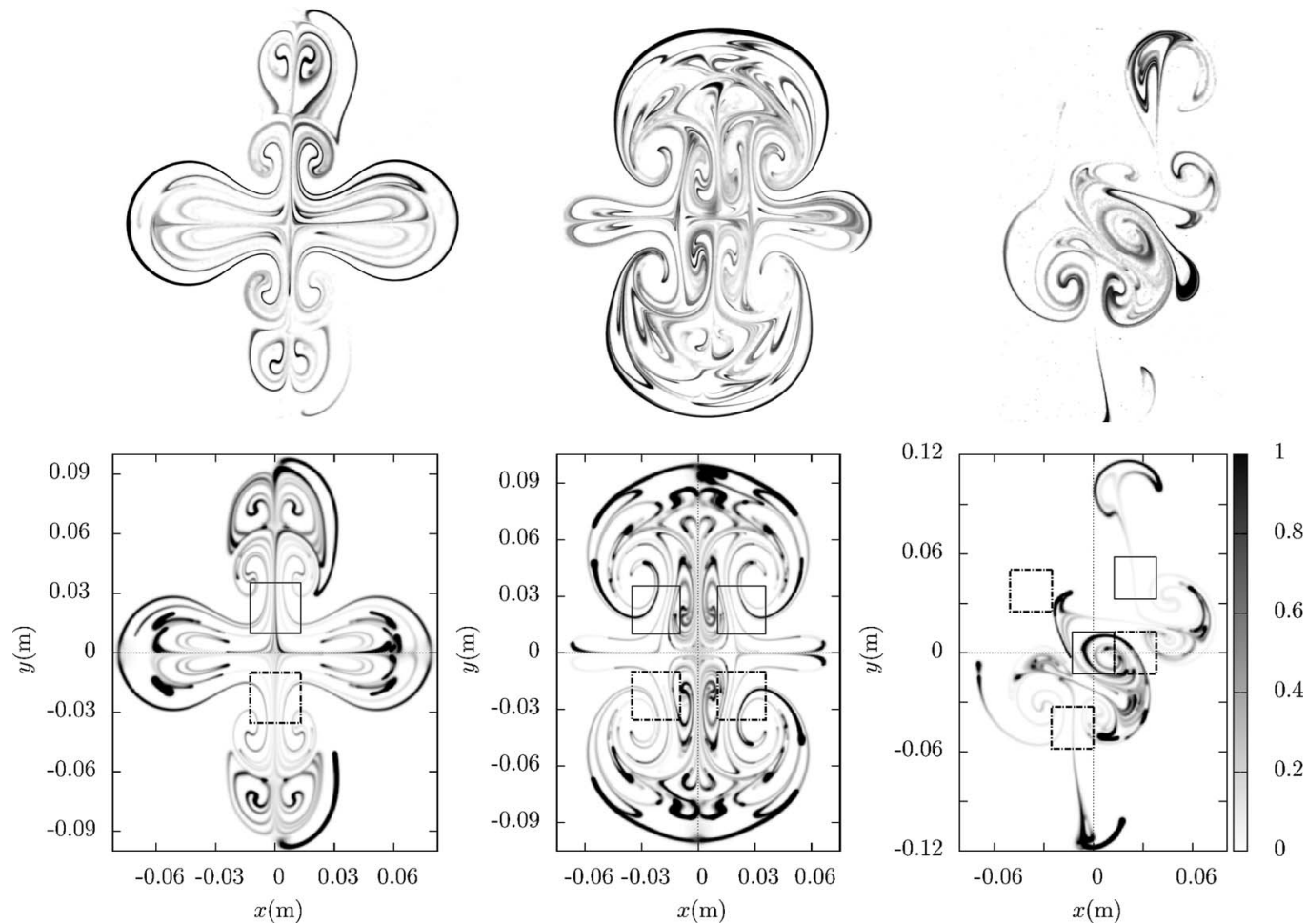

FIG. 3. Oscillating multipole vortex flows. First row, experimental visualization; second row, numerical simulation. First column, quadrupole vortex flow at $t=55 \mathrm{~s}$. Second column, octopole vortex flow at $t=55 \mathrm{~s}$. Third column, disordered vortex flow at $t=25 \mathrm{~s}$. Continuous and dashed squares denote the footprint of magnets polarized in the positive and negative $z$-directions, respectively.

At $t=30 \mathrm{~s}$, most of the scalar is still black because the mixing time has not been reached yet (i.e., the diffusion has not started yet to smear out the filaments). The concentration is slightly weaker in the magnet zone because the filament has been highly stretched and has thus started to diffuse. By contrast, at $t=60 \mathrm{~s}$, some large parts of the filament are grey, in both the numerical and the experimental results. This shows that the DSM is able to predict correctly the concentration as well. It should be noted that the stretching rate $\gamma$ of the filament is close to $0.1 \mathrm{~s}^{-1}$ such that the thickness of the filament, given by the Batchelor scale $\eta_{B}=\sqrt{D / \gamma}$, is of the order of $7 \times 10^{-5} \mathrm{~m}$, i.e., three orders of magnitude smaller than the domain size. To achieve a resolution similar to the one obtained with DSM, a standard numerical method for the integration of the advection-diffusion equation would require a mesh of about $10^{4} \times 10^{4}$ points and therefore, a very large memory. In the present work, DSM used $10^{6}$ points at the end of the calculation, which is easily achievable with any standard computer. Considering that a standard computer can handle $10^{6}$ points, a parallel standard code for the integration of the advection-diffusion equation would require a hundred computers to complete the calculus. In other words, the DSM code is 100 times faster than convectional sequential codes.

At $t=150 \mathrm{~s}$, there is still a good agreement on the position and the intensity of the scalar concentration. It should be mentioned that adjacent filaments start overlapping at some locations, since the DSM constructs the field by adding the concentration from each filament. This can be done due to the linearity of the advection-diffusion equation.

Figure 3 shows the experimental visualization and numerical simulation of the scalar transport in oscillating flows produced by a time-periodic Lorentz force due to different arrays of magnets. The presence of two magnets creates two dipolar vortices oriented alternately in the $x$ and the $y$ direction. This creates four semi-circular patterns with a symmetry in the $x$ and in the $y$ direction. In the presence of four magnets, the experiment exhibits again a symmetric pattern, in good agreement 


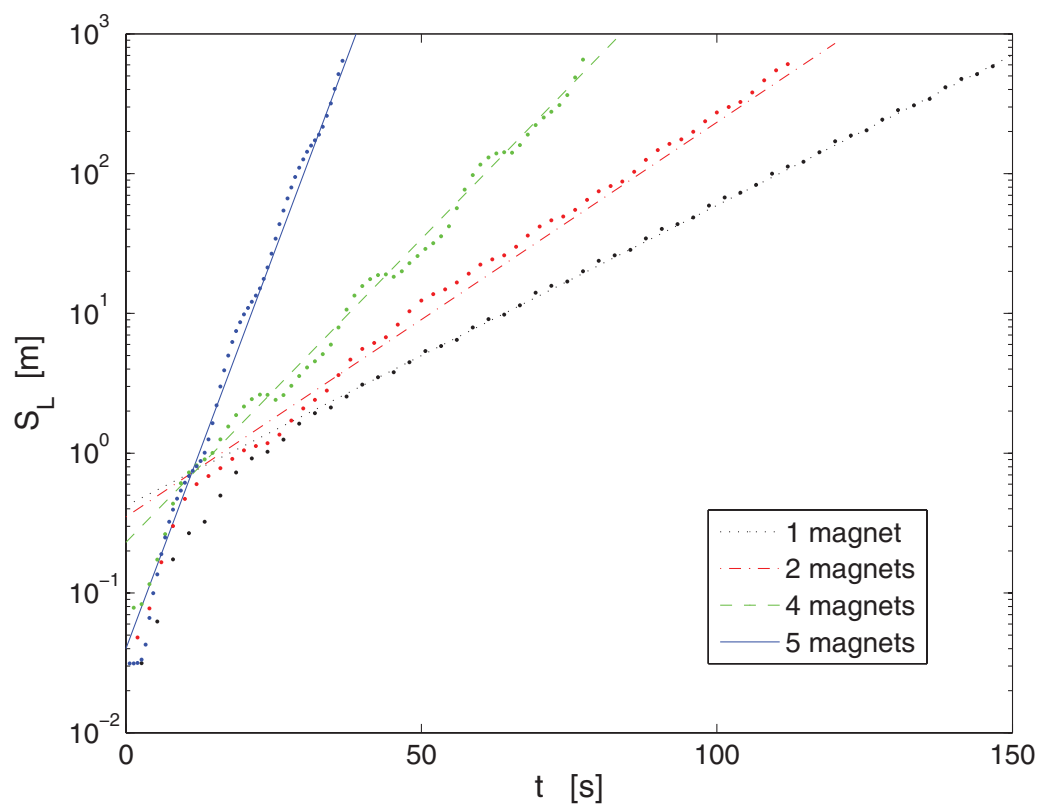

FIG. 4. Total strip length $S_{L}$ as a function of time $t$ for different flows. Dotted line (blue): flow due to one magnet; dasheddotted line (red): flow due to two magnets; dashed line (green): flow due to an ordered array of four magnets; solid line (blue): flow due to a disordered array of five magnets. Symbols correspond to the numerically calculated length and lines correspond to an exponential fit using (11). The values of $t_{0}$ used in the fittings are $t_{0}=-54 \mathrm{~s}$ for 1 magnet, $t_{0}=-33 \mathrm{~s}$ for 2 magnets, $t_{0}=-25 \mathrm{~s}$ for 4 magnets, and $t_{0}=-1.5 \mathrm{~s}$ for 5 magnets.

with the numerical result. Finally, for five disordered magnets, the scalar rolls-up inside dipolar structures whose orientations seem random but are in fact coherent between the numerics and the experiment. However, there are some differences in some places due to the high sensitivity to the initial conditions.

When the number of magnets increases, the scalar is stretched more quickly and it fills a larger area (note the earlier time and the larger domain size for five magnets). This higher stretching rate also accelerates the diffusion at the center of the domain. However, there are still some dark filaments on the outer parts, which reveal a very weak stretching. This non-uniform stretching of the filament is due to the localization of the flow around the magnets. It will be interesting to see whether this non-homogeneous effect creates any differences compared with the homogeneous models described in the following.

\section{B. Stretching rate}

Together with the diffusion of the scalar, the DSM method allows to compute the total length of the strip $S_{L}$ as it is convected by the flow. This is interesting because it is the stretching rate which governs the thickness and the diffusion time of the filament and, as will be seen, its overall concentration statistics. It should be noted that the detailed kinematics of the strip line is very sensitive to the location of the initial dye blob. However, the total strip length $S_{L}$ grows exponentially with almost the same growth rate when the blob is initialized in a high stretching region $(x \approx 0, y \approx 0)$.

Figure 4 shows the length of the strip as a function of time for different flows. As the flow evolves, the strip is stretched and folded so that its length tends to grow exponentially from $3 \mathrm{~cm}$ to $20 \mathrm{~m}$ in approximately 8 cycles $(t=160 \mathrm{~s})$ for the dipolar flow, and in only 1 cycle $(t=20 \mathrm{~s})$ for the disordered flow. This exponential growth in time of $S_{L}$ by stretching and folding is indicative of chaotic mixing. ${ }^{25}$ The length $S_{L}$ is well fitted at late stages by an exponential law

$$
S_{L}=S_{0} e^{\gamma\left(t-t_{0}\right)},
$$




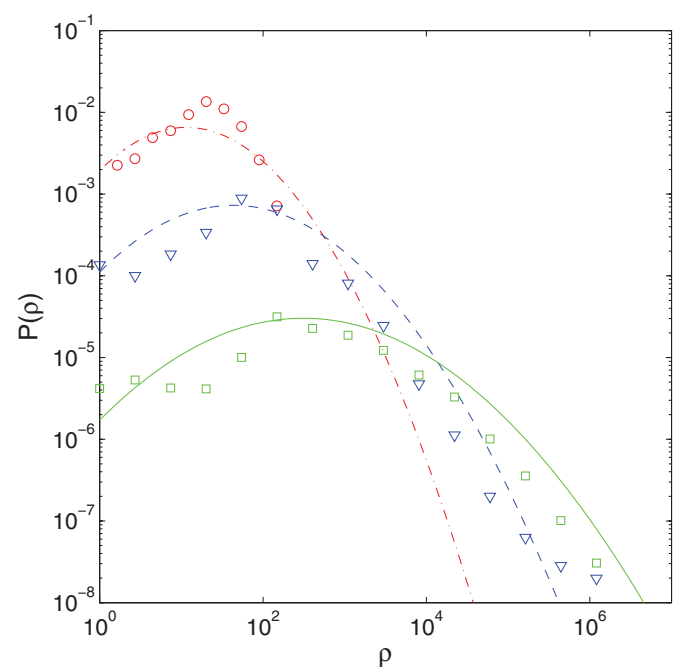

(a)

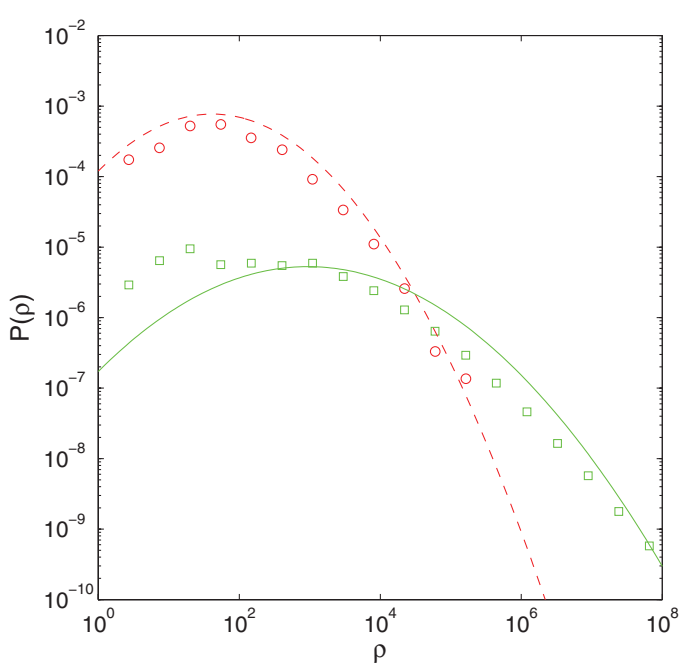

(b)

FIG. 5. Normalized PDFs of elongation of the strip. In (a) the flow is created by one magnet and taken at $t=20 \mathrm{~s}(\mathrm{o}$, dashed-dotted line, red), $t=60 \mathrm{~s}(\nabla$, dashed line, blue), and $t=120 \mathrm{~s}$ ( $\square$, solid line, green). In (b) the flow is created by five magnets and taken at $t=20 \mathrm{~s} \mathrm{(o,} \mathrm{dashed} \mathrm{line,} \mathrm{red)} \mathrm{and} t=37 \mathrm{~s}$ ( $\square$, solid line, green). Lines correspond to the theoretical prediction (12) where $\gamma=0.05$ in (a) and $\gamma=0.26$ in (b) come from the fit of the total strip length of Fig. 4.

which is plotted in Fig. 4 as lines. The temporal delay $t_{0}$ allows to take into account the transient stage, where the strip length increases very quickly. This is especially visible for 1 magnet (before $t=30 \mathrm{~s}$ ) and for 2 magnets (before $t=20 \mathrm{~s}$ ). This is probably due to the fact that the dye is initially located at the center where the stretching rate is much larger than the average stretching rate within the whole cavity.

Random multi-step stretchings produce a log-normal distribution for the probability of the stretching intensity, a property applied to mixing in random flows by Kalda. ${ }^{33}$ This probability $P(\rho, \mathrm{t})$ is defined as the probability on the strip at time $t$ that a point of the strip has been stretched by a factor $\rho$. Moreover, Meunier and Villermaux ${ }^{24}$ showed that the average of $\log \rho$ increases twice faster than its variance in 2D (and three times faster in 3D). This fixes the coefficients of the log-normal law, which only depends on $\gamma$

$$
P(\rho)=\frac{e^{-\gamma\left(t-t_{0}\right)}}{\sqrt{4 \pi \gamma\left(t-t_{0}\right) / 3}} \exp \left[-\frac{\left(\log \rho-2 \gamma\left(t-t_{0}\right) / 3\right)^{2}}{4 \gamma\left(t-t_{0}\right) / 3}\right] .
$$

Here, $\gamma$ corresponds to the mean stretching rate which has been measured from Fig. 4. These predictions are plotted in Fig. 5 and compared to the numerical results. It is clear that the numerical PDFs of stretching rates are indeed close to log-normal and there is an excellent agreement with the theoretical prediction, especially since there is no fitting parameter. This validates the mechanism of a random multi-step stretching of the filament. It is interesting to note that the heterogeneity of the flow in that case has almost no influence on the nature of the PDF of stretching, whose log-normal shape is attractive. The only effect of the heterogeneity is to accelerate the stretching in the early stages but the broadening of the distributions is still proportional to the mean stretching rate with the same multiplying factor.

\section{Probability density functions}

In order to quantify the mixing efficiency, we use the histogram or probability density function $P(c)$ of the concentration levels $c . P(c) d c$ is defined as the normalized number of pixels whose concentration is in the interval $[c, c+d c]$. Figure 6(a) shows the PDF measured experimentally from the visualizations of the dipolar flow at several times. The PDF exhibits the well known U-shape 


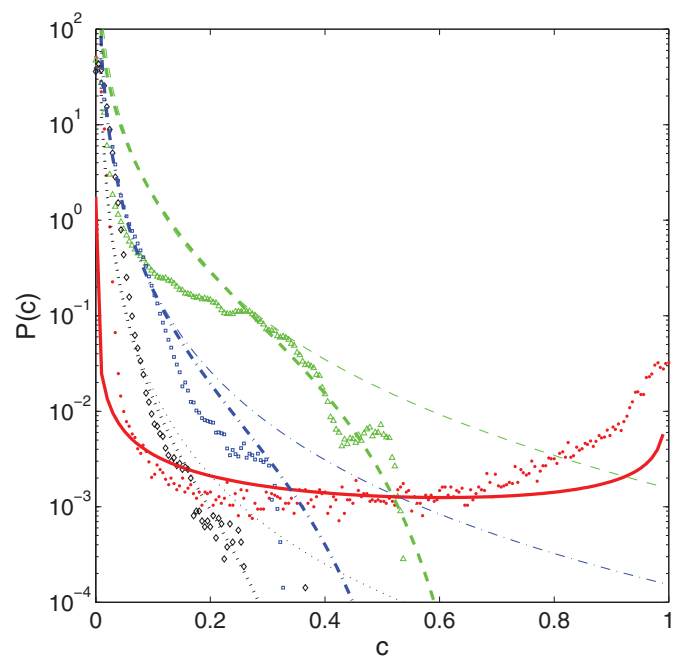

(a)

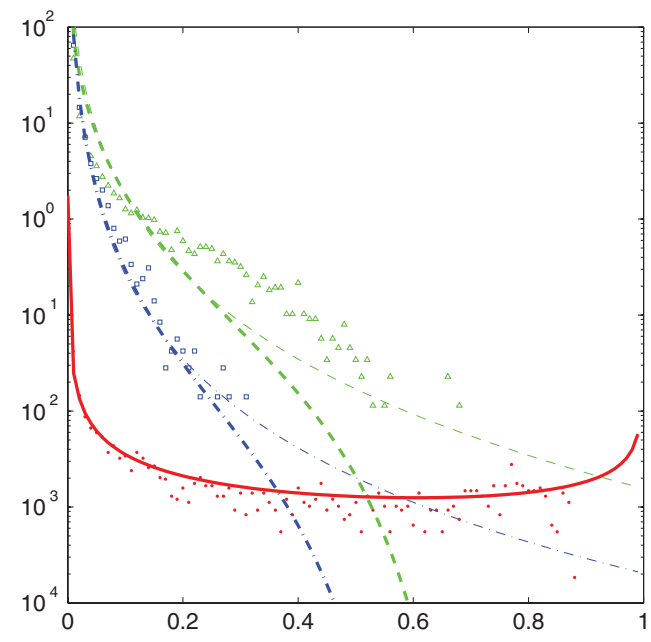

(b)

FIG. 6. Normalized PDFs of the scalar distributions obtained (a) experimentally and (b) numerically for the flow created by 1 magnet at $t=0 \mathrm{~s}(\bullet$, solid line, red), $t=60 \mathrm{~s}$ ( $\triangle$, dashed line, green), $t=120 \mathrm{~s}$ ( $\square$, dashed-dotted line, blue), and $t=300 \mathrm{~s}$ $\left(\diamond\right.$, dotted line, black). Thick lines correspond to the theoretical prediction (14) with $\gamma=0.05 \mathrm{~s}^{-1}$ given by Fig. 4 and $s_{0}=0.01 \mathrm{~cm}$. Thin lines correspond to the approximation (16) at late stages.

at the initial state ( $t=0 \mathrm{~s}$, red symbols). It comes from the large number of pixels of the background $(c=0)$ and the moderate number of pixels in the blob with the maximal initial concentration $(c=1)$. At $t=60 \mathrm{~s}$ (green symbols), the shape of the PDF is completely different. There are no pixels with a concentration larger than 0.6, which means that all parts of the filaments have reached the mixing time at which diffusion starts. The PDF is a decreasing function of the concentration because the highest concentration, corresponding to the lowest stretching rate, is rare. As time evolves (from $t=120 \mathrm{~s}$ to $t=300 \mathrm{~s}$ ), the PDF decreases quicker since the maximum concentration decreases due to diffusion.

Figure 6 shows that the experimental observations (Fig. 6(a)) and numerical DSM calculations (Fig. 6(b)) display the same general trend as that predicted by the theory from Eqs. (14) and (16). Specifically, the PDF changes from U-shaped to decreasing functions as time evolves. Note that large stages could not be calculated numerically because the length of the filament and thus the number of points was too large.

Figure 7 shows the PDF of concentration found for a disordered array of five magnets. The same trend is obtained numerically and experimentally, which highlights the fact that the disorder of the flow does not modify the properties of the PDF. It should be noted that it is the first time that experimental PDFs are compared to numerical PDFs at such a large Péclet number and for a complex flow. This is possible here because the flow can be calculated numerically thanks to a good knowledge of the electromagnetic forcing and because the weak diffusion is calculated using the DSM method.

The advantage of the numerical result is that the PDF of stretching rate of the filament is known. The model developed in Sec. IV B can thus be used to predict the PDF of concentration. Indeed, in the absence of aggregation between two adjacent filaments, the PDF of a piece of filament is simply related to its stretching factor $\rho$. Indeed, the profile of concentration is Gaussian and given by (10) with dimensionless times given by

$$
\xi(\rho)=\frac{D t}{s_{0}^{2}} \frac{\rho^{2}-1}{\log \rho}
$$

if the stretching rate is constant in time. This hypothesis allows to reconstruct the PDF of concentration assuming that the PDF of stretching rate is given by (12). Summing over all stretching rates 


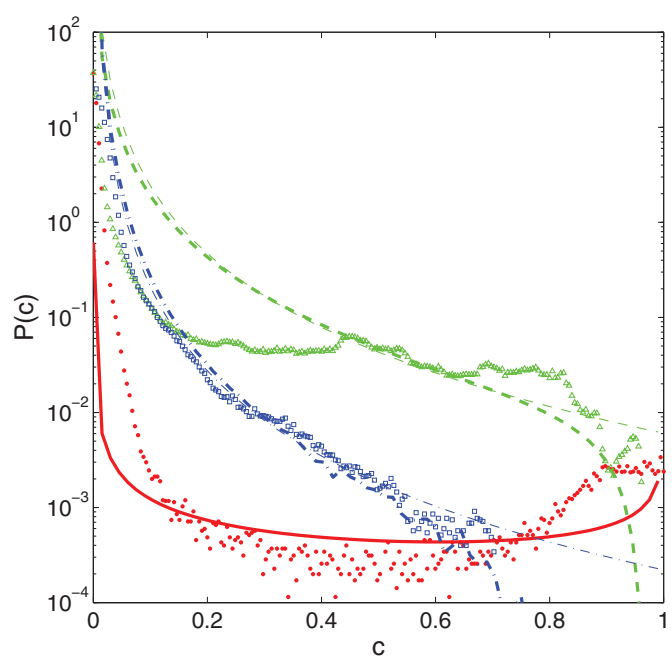

(a)

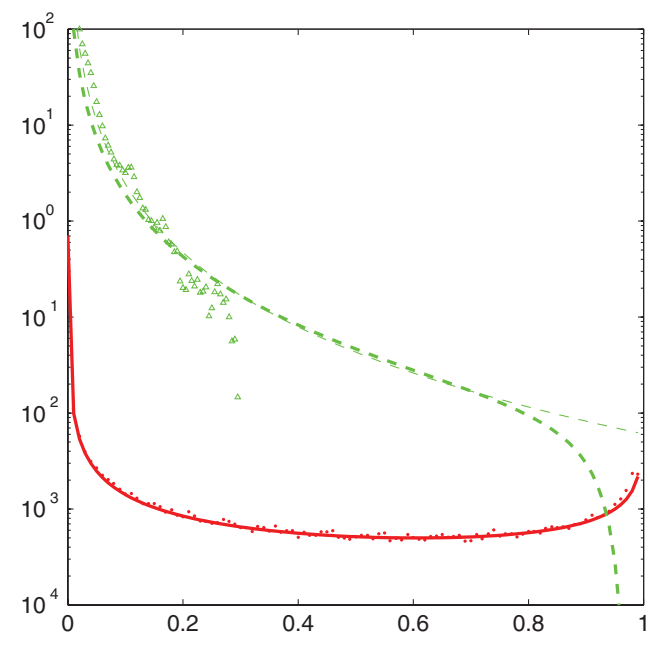

(b)

FIG. 7. Normalized PDFs of the scalar distributions obtained experimentally (a) and numerically (b) for the flow created by five disordered magnets at $t=0 \mathrm{~s}(\bullet$, solid line, red), $t=20 \mathrm{~s}(\triangle$, dashed line, green), and $t=120 \mathrm{~s}$ ( $\square$, dashed-dotted line, blue). Thick lines correspond to the theoretical prediction (14) with $\gamma=0.26 \mathrm{~s}^{-1}$ given by Fig. 4 and $s_{0}=0.025 \mathrm{~cm}$. Thin lines correspond to the approximation (16) at late stages.

leads to 24

$$
P(c)=\frac{A}{c} \int_{4 \xi(\rho)<c^{-2}-1} \sqrt{\frac{1+4 \xi(\rho)}{-\log (c \sqrt{1+4 \xi(\rho)})}} e^{-\frac{(\log \rho-2 \gamma t / 3)^{2}}{4 \gamma / t 3}} \frac{d \rho}{\rho},
$$

where $A$ is the normalization factor. This solution is plotted in Figs. 6 and 7 as thick solid lines for all times. It is in reasonable agreement with the experimental and the numerical results. It must be remembered that the stretching rate $\gamma$ is not a fitting parameter, but is given by the value obtained numerically for the exponential growth of the length of the filament.

This exact solution can be approximated at late stages, when the filament has been highly stretched. Indeed, for large stretching factors $\rho$, the dimensionless time given by (13) can be approximated by $\xi \approx D t \rho^{2} / s_{0}^{2}$ such that the formula for the PDF can be simplified using the change of variable $\zeta=-\log \rho-\log \left(2 c \sqrt{D t} / s_{0}\right)$ :

$$
P(c)=\frac{2 A \sqrt{D t}}{c s_{0}} \int_{0}^{+\infty} \exp \left[-\frac{\left(\zeta+4 \gamma t / 3+\log \left(2 c \sqrt{D t} / s_{0}\right)\right)^{2}}{4 \gamma t / 3}\right] \frac{d \zeta}{\sqrt{\zeta}} .
$$

At late stages, the term in the Gaussian $\log \left(2 c \sqrt{D t} / s_{0}\right)+4 \gamma t / 3$ is large such that the integral can be approximated by the value of the Gaussian at $\zeta=0$. This leads to a log-normal form of the PDF of concentration:

$$
P(c) \approx \frac{1}{c} \exp \left[-\frac{\left(-\log c-4 \gamma t / 3-\log \left(2 \sqrt{D t} / s_{0}\right)\right)^{2}}{4 \gamma t / 3}\right] .
$$

This approximation is plotted in Figs. 6 and 7 as thin dashed lines. It is very close to the exact solution for small concentration $c$ because it corresponds to large stretching rates. However, there is a clear disagreement for $c$ close to unity because it comes from parts of the filament with a very weak stretching rate. 
It should be noted that this approximation corresponds exactly to the PDF $Q\left(c_{\max }\right)$ of the maximum concentration ${ }^{19}$ (i.e., the maximal concentration of the spatial profile transverse to the filament, at its center). Indeed, if $\xi \approx D t \rho^{2} / s_{0}^{2}$ then $c_{\max } \approx s_{0} / 2 \rho \sqrt{D t}$ such that $Q\left(c_{\max }\right)=P(\rho) d \rho / d c_{\max }$ is also $\log$-normal with the same parameters as in (16) if $1 / c_{\max }^{2}$ is replaced by $\exp \left(-\log \left(c_{\max }\right)\right) / c_{\max }$.

\section{DISCUSSION AND CONCLUSIONS}

We have analyzed experimentally and theoretically the mixing in laminar time-periodic vortex flows in a shallow electrolytic layer. The flow patterns were generated by the interaction of multipolar magnetic field distributions produced by arrays of magnets and a uniform alternating current. First, using the flow fields calculated numerically through the Q2D model, the Lagrangian particle tracking of different flows was compared with experimental visualization using dye. It was found that the mixing produced by electromagnetically driven flows is strongly influenced by the magnetic field distributions used. With a single magnet or ordered arrays of two and four magnets, clear symmetry lines appear in the flow patterns. The displayed flow symmetries, acting as mixing inhibitors, are originated by symmetric Lorentz forces that, in turn, appear as a consequence of inherent symmetries in the magnetic field distribution. These symmetries are broken when a non-symmetric magnetic field distribution is used, as the one given by a disordered array of magnets. It is important to note that the Q2D model that introduces a linear friction with the bottom wall leads to satisfactory results.

On the other hand, the advection-diffusion transport of a scalar was addressed by implementing the diffusive strip method. ${ }^{24}$ In this new model, the position of an advected material strip is computed kinematically, and the associated advection-diffusion problem is solved by using the computed local stretching rate along the strip, assuming that the diffusing strip thickness is smaller than its local radius of curvature. This widely legitimate assumption reduces the numerical problem to the computation of a single variable along the strip, thus making the method extremely fast and applicable to high Péclet numbers. This numerical method makes the link between the standard fluid dynamics simulation methods which are limited to small Péclet numbers and the Lagrangian tracking methods which do not model the diffusion of a scalar. The DSM model correctly captures the main physical features of the scalar mixing in electromagnetically driven flows in multipolar magnetic fields. In general, a good qualitative comparison is found between numerical results and experimental observations. Furthermore, as the strip is stretched and folded, the method allows to calculate the total length of the strip which was found to grow exponentially, indicating that standard chaotic mixing is realized in flows. The main advantage of this numerical method is that the PDF of stretching factors can be measured directly without any assumption. They are very close to log-normal as was found for homogeneous turbulent mixing in the Batchelor regime. ${ }^{33}$ The variance and the mean stretching rate increase linearly in time, with the variance being twice larger than the mean, a feature of $2 \mathrm{D}$ flows. ${ }^{24}$ This relation reduces the model to a single parameter, the total stretching rate of the filament, which can be measured directly in the simulations (rather than being a fitting parameter).

The PDFs of concentration were also measured from the experimental and the numerical results. They are in fair agreement, with a U-shape at early stages and a decreasing behavior at late stages. A theoretical model based on the log-normal PDF of stretching factors shows also a correct agreement with both results. The U-shape of the concentration PDF at early stages comes from the Gaussian transverse concentration profile of the filament, which leads to a PDF equal to $1 / c \log (c)$. The decreasing behavior at late stages is in fact half a log-normal law, which can be recovered asymptotically for large stretching factors. Indeed, the maximum concentration $c_{\max }$ of a filament which has been stretched by $\rho$ is equal to $1 / \rho$ such that $\log c_{\max }$ is normal if $\log \rho$ is normal. It is surprising to see that this idealized model developed for homogeneous turbulence is still valid for an organized and symmetric flow such as the oscillating dipole or quadrupole.

Due to the statistical nature of the information required for the present investigation, it is difficult to establish what the uncertainties in the experimental observations or numerical calculation are. However, it is encouraging that the predictions of DSM theory coincide qualitatively and in many instances quantitatively with the experimental and numerical data. The results presented indicate that the DSM was useful for the analysis of a quasi-2D large Péclet number flow generated by localized dipoles. Moreover, it does not require a large amount of memory since it calculates a 1D 
filament instead of a 2D distribution. This is why this method will probably be even more useful for 3D mixing problems, where sheets of scalars are stretched and folded. This extension of the method might be technically difficult but conceptually easy since the thickness of the diffusing sheet verifies the same law as in two dimensions. The results of the present study, added to those already available for flows in porous media ${ }^{34}$ indicate that the DSM can be useful in the analysis of flows where the scalar diffuses much less than the momentum.

However, the main message of this paper is that the PDF of stretching factors and the PDF of concentration are directly related to the mean stretching rate of the filament. The simple measure of the length of the filament with time can thus give predictions for both PDFs thanks to the simple model developed for turbulent flows.

\section{ACKNOWLEDGMENTS}

A. Figueroa thanks a mobility grant from the SCAT project and the ALFA Programme of EuropeAid during the stay at IRPHE. A. Figueroa also thanks CONACYT and PROMEP for support via doctoral and postdoctoral fellowships, respectively. Support from CONACYT under Project No. 131399 is thankfully acknowledged.

${ }^{1}$ W. L. Chien, H. Rising, and J. M. Ottino, "Laminar mixing and chaotic mixing in several cavity flows," J. Fluid Mech. 170, 355 (1986).

${ }^{2}$ H. Aref and S. Balachandar, "Chaotic advection in a Stokes flow," Phys. Fluids 29, 3515-3521 (1986).

${ }^{3}$ J. Sommeria, "Electrically driven vortices in a strong magnetic field," J. Fluid Mech. 189, 553-569 (1988).

${ }^{4}$ G. A. Voth, T. C. Saint, G. Dobler, and J. P. Gollub, "Mixing rates and symmetry breaking in two-dimensional chaotic flow," Phys. Fluids 15, 2560-2566 (2003).

${ }^{5}$ L. Rossi and S. Lardeau, "Lamination and folding in electromagnetically driven flows of specified geometries," J. Turbul. 12, 1-31 (2011).

${ }^{6}$ L. Rossi, D. Doorly, and D. Kustrin, "Lamination and mixing in three fundamental flow sequences driven by electromagnetic body forces,” Phys. Rev. E 86, 026313 (2012).

${ }^{7}$ A. Figueroa, F. Demiaux, S. Cuevas, and E. Ramos, "Electrically driven vortices in a weak dipolar magnetic field in a shallow electrolytic layer," J. Fluid Mech. 641, 245-261 (2009).

${ }^{8}$ A. Figueroa, S. Cuevas, and E. Ramos, "Electromagnetically driven oscillatory shallow layer flow," Phys. Fluids 23, 013601 (2011).

${ }^{9}$ A. Figueroa, "Dynamics of electromagnetically driven vortices," Doctoral thesis (National Autonomous University of Mexico, 2010)

${ }^{10}$ J. C. McWilliams, Fundamentals of Geophysical Fluid Dynamics (Cambridge University Press, Cambridge, 2006).

${ }^{11}$ C. A. Brown, G. A. Jackson, and D. A. Brooks, "Particle transport through a narrow tidal inlet due to tidal forcing and implications for larval transport,” J. Geophys. Res. 105, 141-156, doi:10.1029/2000JC000211 (2000).

${ }^{12}$ E. Wolanski, E. Drew, K. M. Abel, and J. O'Brien, "Tidal jets, nutrient upwelling and their influence on the productivity of the alga Halimeda in the Ribbon Reefs, Great Barrier Reef," Est. Coast. Shelf Sci. 26, 169-201 (1988).

${ }^{13}$ H. Aref, "Stirring by chaotic advection," J. Fluid Mech. 143, 1-21 (1984).

${ }^{14}$ H. Aref, "The development of chaotic advection," Phys. Fluids 14, 1315-1325 (2002).

${ }^{15}$ C. W. Leong and J. M. Ottino, "Experiments on mixing due to chaotic advection in a cavity," J. Fluid Mech. 209, $463-499$ (1989).

${ }^{16}$ P. V. Danckwerts, "The definition and measurement of some characteristics of mixtures," Appl. Sci. Res. A 3, 279-296 (1952).

${ }^{17}$ D. Rothstein, E. Henry, and J. P. Gollub, "Persistent patterns in transient chaotic fluid mixing," Nature 401, 770-772 (1999).

${ }^{18}$ D. R. Fereday, P. H. Haynes, and J. C. Vassilicos, "Scalar variance decay in chaotic advection and Batchelor-regime turbulence," Phys. Rev. E 65, 035301 (2002).

${ }^{19}$ P. Meunier and E. Villermaux, "How vortices mix,” J. Fluid Mech. 476, 213-222 (2003).

${ }^{20}$ J. Duplat and E. Villermaux, "Mixing by random stirring in confined mixtures," J. Fluid Mech. 617, 51-86 (2008).

${ }^{21}$ M. Giona, A. Adrover, S. Cerbelli, and V. Vitacolonna, "Spectral properties and transport mechanisms of partially chaotic bounded flows in the presence of diffusion," Phys. Rev. Lett. 92, 114101 (2004).

${ }^{22}$ E. Gouillart, N. Kuncio, O. Dauchot, B. Dubrulle, S. Roux, and J.-L. Thiffeault, "Walls inhibit chaotic mixing," Phys. Rev. Lett. 99, 114501 (2007).

${ }^{23}$ E. Villermaux, "On dissipation in stirred mixtures," Adv. Appl. Mech. 45, 91-107 (2012).

${ }^{24}$ P. Meunier and E. Villermaux, "The diffusive strip method for scalar mixing in two-dimensions," J. Fluid Mech. 662 , 134-172 (2010).

${ }^{25}$ J. M. Ottino, The Kinematics of Mixing: Stretching, Chaos, and Transport (Cambridge University Press, Cambridge, 1989).

${ }^{26}$ J. P. Gleeson, "Transient micromixing: Examples of laminar and chaotic stirring,” Phys. Fluids 17, 100614 (2005).

${ }^{27}$ T. Solomon, J. Winokur, G. O'Malley, and M. Paoletti, "Chaotic mixing and superdiffusion in a two-dimensional array of vortices," Bull. Am. Phys. Soc. 53(15), 256 (2008), Abstract only. 
${ }^{28}$ N. Ouellette and J. P. Gollub, “Curvature fields, topology, and the dynamics of spatiotemporal chaos,” Phys. Rev. Lett. 99, 194502 (2007).

${ }^{29}$ R. A. D. Akkermans, L. P. J. Kamp, H. J. H. Clercx, and G. J. F. van Heijst, “Three-dimensional flow in electromagnetically driven shallow two-layer fluids," Phys. Rev. E 82, 026314 (2010).

${ }^{30}$ S. Cuevas, S. Smolentsev, and M. Abdou, "On the flow past a magnetic obstacle," J. Fluid Mech. 553, 227-252 (2006).

${ }^{31}$ M. McCaig, Permanent Magnets in Theory and Practice (Wiley, New York, 1977).

${ }^{32}$ M. Griebel, T. Dornseifer, and T. Neunhoeffer, Numerical Simulation in Fluid Dynamics (SIAM, New York, 1998).

${ }^{33}$ J. Kalda, "Simple model of intermittent passive scalar turbulence," Phys. Rev. Lett. 84, 471-474 (2000).

${ }^{34}$ T. Le Borgne, M. Dentz, and E. Villermaux, "Stretching, coalescence, and mixing in porous media," Phys. Rev. Lett. 110, 204501 (2013). 